\title{
Discrete hopping model of exciton transport in disordered media
}

\author{
V. M. Burlakov, K. Kawata, H. E. Assender, and G. A. D. Briggs \\ Department of Materials, University of Oxford, Parks Road, Oxford OX1 3PH, United Kingdom
}

A. Ruseckas and I. D. W. Samuel

School of Physics and Astronomy, University of St. Andrews, St. Andrews KY16 9SS, United Kingdom

(Received 16 March 2005; revised manuscript received 14 June 2005; published 12 August 2005)

\begin{abstract}
A model of dispersive exciton transport has been developed for a medium with exciton energy levels randomly distributed in both space and energy scale. For a boxcar density of states of excitons an analytical solution is given describing the exciton density as a function of time and the proximity to the exciton quenching interfaces. The model parameters, such as exciton lifetime, effective number of exciton energy levels within inhomogeneously broadened density of states, and hopping distance and hopping rate constants, could be determined using time-resolved photoluminescence data. The developed model is verified via comparison with experimental data on the time-dependent photoluminescence decay of the conjugated polymer MEH-PPV and on the spectrum of internal quantum efficiency of the heterojunction photovoltaic device based on the $\mathrm{MEH}-\mathrm{PPV} / \mathrm{TiO}_{2}$ nanostructure.
\end{abstract}

DOI: 10.1103/PhysRevB.72.075206

PACS number(s): 71.35.-y, 78.55.Kz

\section{INTRODUCTION}

A growing demand in low-cost electro-optical and photovoltaic devices has resulted in intensive studies of amorphous organic materials, such as conjugated polymers. Effective transport of excitons in conjugated polymers is extremely important for performances of organic lightemitting diodes ${ }^{1}$ and of plastic excitonic solar cells. ${ }^{2} \mathrm{~A}$ crucial step in the photovoltaic process, for instance, is the conversion of photogenerated excitons into charge carriers at the polymer-inorganic interfaces. High quantum yield of charge carriers could be achieved if the excitons would travel far enough from their generation points to appropriate interfaces where they can dissociate, injecting electrons into the electrode. The holes remaining in the polymer diffuse to the opposite electrode, completing charge separation.

Only a fraction of the photogenerated excitons reach relevant interfaces while many of them decay by emitting light or exciting vibrations of the polymer molecules. Besides a limited lifetime, the length scale of the exciton migration is restricted by the spatial dependence of the exciton energyi.e., inhomogeneous broadening of exciton energy level. A conjugated polymer chain, for example, can be thought of as a series of molecular segments linked with each other at topological faults. Each segment has certain lowest unoccupied molecular orbital (LUMO) and highest occupied molecular orbital (HOMO) levels depending in part on its conjugation length. While migrating, excitons on average lose their energy by predominantly hopping to lower-energy sites. Therefore the migration of excitons slows down when they reach the low-energy sites where they find fewer sites with lower energy in its neighborhood. ${ }^{3-5}$ Due to such dispersive migration, the exciton diffusion cannot be described using a constant diffusion coefficient, but a time-dependent one. Usually such a process of migration of species in a random media is modeled using a Monte Carlo method ${ }^{6}$ or by solving a master equation. ${ }^{7}$ There are also a number of analytical approaches addressing highly nonequilibrium (dispersive) transport of excitons and charge carriers in disordered media (see, for example, Refs. 8 and 9 and references therein).

In the present article we develop a simple discrete kinetic model, which accounts for energetic inhomogeneity and allows a quantitative description of the exciton migration taking into account their dissociation at the interfaces with an inorganic semiconductor $\left(\mathrm{TiO}_{2}\right)$. The model containing few fitting parameters is verified by calculating the timedependent photoluminescence of excitons in MEH-PPV and absorbed photon-to-conducting-electron conversion efficiency (APCE) of solar devices based on the conjugated polymer- $\mathrm{TiO}_{2}$ composite and comparing the results with experimental data.

\section{MODEL FOR EXCITON TRANSPORT}

The hopping mechanism of excitons is considered to be a dipole-dipole resonance energy transfer known as the Förster mechanism. ${ }^{3-5,10,11}$ According to this mechanism the exciton hops to a neighboring site with the energy level closest to the initial one. Due to structural relaxation around the exciton in a real system, its energy decreases after hopping; therefore, the exciton loses its energy during migration. Taking into account the coupling between exciton migrations in space with a continuous decrease in their energy we assume that the model shown in Fig. 1(a) would describe exciton transport in disordered systems, such as conjugated polymers. According to Fig. 1 the system is modeled by a onedimensional (1D) lattice with lattice sites each representing a slice of the system perpendicular $x$ direction. Each lattice site in Fig. 1(a) contains one and the same set of energy levels, which number reflects an average number of hops an exciton makes along one direction before it becomes localized. Exciton migration within each slice is not considered, as migrations in the $x, y$, and $z$ directions are independent and the energy levels can be assumed to be equidistant for simplicity, suggesting a boxcar shape for the exciton density of states. 
(a)

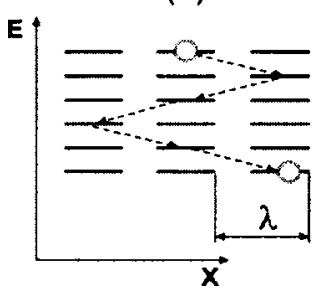

(b)

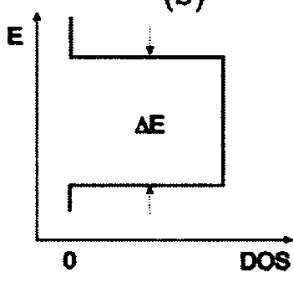

FIG. 1. (a) Illustration of exciton migration (arrows) in a onedimensional lattice featuring a system with positional and energetic disorder of exciton energy levels. Each lattice site contains identical set of descrete energy levels, and the lattice constant of the model system is taken to be equal to an average hopping distance $\lambda$ of excitons in disordered system. (b) Exciton density of states (DOS) in the model system.

The resonance character of the exciton hopping probability is taken into account by allowing the excitons to hop only to the next lower-energy level, and the average hopping distance $\lambda$ is preserved by exciton hopping only to the nearestneighboring site.

Depending on the dimensionality of the real system the sites in the model system could be arranged in a 1D chain, square lattice, or a cubic lattice. Note that the lattice symmetry would not bias the transport properties, as we assign a set of energy levels to each lattice site. We illustrate the potential of the model system with an example 1D chain, such as shown in Fig. 2. The time evolution of the probability $p_{i j}(t)$ for an exciton to be at lattice site $j$ and at energy level $i$ is given by

$$
\frac{d p_{i, j}(t)}{d t}=\frac{\gamma}{2}\left[p_{i-1 j-1}(t)+p_{i-1 j+1}(t)\right]-\left(\gamma+\tau^{-1}\right) p_{i, j}(t),
$$

where $\gamma$ is hopping probability and $\tau$ is the exciton lifetime due to spontaneous recombination processes. An exciton could also dissociate at the interface with inorganic semiconductor. A general solution for Eq. (1) can be sought in the form

$$
p_{i, j}(t)=w_{j, i} P_{i}(t),
$$

where $P_{i}(t)$ is an average probability of finding an exciton on the $i$ th energy level and $w_{j, i}$ represents the probability for the exciton to be photogenerated at a distance of $j$ lattice sites

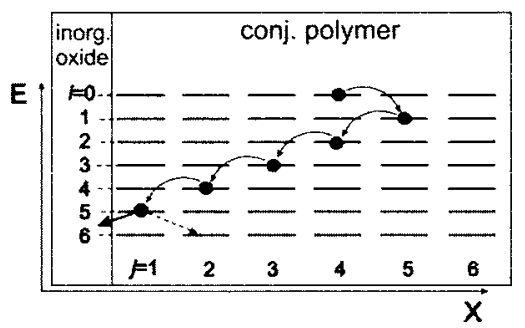

FIG. 2. Exciton (circle) photogenerated on energy level $i=0$ migrating either by reaching the lowest-energy level (dashed arrow) or by reaching the interface at which it is dissociated by injecting an electron into inorganic oxide (thick arrow). from the interface to avoid dissociation at this interface after $i$ hops.

If the exciton generation is homogeneous in space (independent of $j$ ), then far from any interfaces the distribution of excitons is also homogeneous-i.e., $w_{j, i}=$ const. The timedependent exciton population is then given by the probability $P_{i}(t)$ described according to Eqs. (1) and (2):

$$
\begin{gathered}
\frac{d P_{0}(t)}{d t}=-\left(\gamma+\tau^{-1}\right) P_{0}(t), \\
\frac{d P_{i}(t)}{d t}=\gamma P_{i-1}(t)-\left(\gamma+\tau^{-1}\right) P_{i}(t), \quad i=1,2, \ldots, n-1, \\
\frac{d P_{n}(t)}{d t}=\gamma P_{n-1}(t)-\tau^{-1} P_{n}(t) .
\end{gathered}
$$

Using for simplicity the initial conditions $P_{0}(0)=1, P_{i}(0)$ $=0, i=1,2, \ldots, n-1$, one can obtain the solution of Eqs. (2) and (3) in the form of the Poisson distribution of exciton density over $n$ energy levels:

$$
\begin{gathered}
P_{i}(t)=\frac{\gamma^{i} t^{i}}{i !} e^{-\gamma t} e^{-t / \tau}, \quad i=0,1, \ldots, n-1, \\
P_{n}(t)=\left\{1-\sum_{i=0}^{n-1} P_{i}(t)\right\} e^{-t / \tau},
\end{gathered}
$$

The time-dependent diffusion coefficient for excitons, $D(t)$, is defined as

$$
D(t)=\frac{1}{2} \frac{d\left\langle l^{2}\right\rangle}{d t} .
$$

The square diffusion distance $\left\langle l^{2}\right\rangle$ in the $1 \mathrm{D}$ case can be defined by considering average excess number of hops made by exciton in the directions "+" or "-" during the total number $m=n_{+}+n_{-}$of hops multiplied by the square of the hopping distance $\lambda$ :

$$
\begin{aligned}
\left\langle l^{2}\right\rangle= & \lambda^{2}\left\langle\left(n_{+}-n_{-}\right)^{2}\right\rangle=\lambda^{2}\left\langle\left(2 n_{+}-m\right)^{2}\right\rangle \\
= & \lambda^{2} \sum_{k=0}^{m}\left(\frac { m ! } { n _ { + } ! ( m - n _ { + } ) ! } \left(2 n_{+}\right.\right. \\
& \left.-m)^{2}\right) / \sum_{k=0}^{m}\left(\frac{m !}{n_{+} !\left(m-n_{+}\right) !}\right) .
\end{aligned}
$$

By direct calculations it can be shown that for $m \geqslant 4$

$$
\left\langle\left(n_{+}-n_{-}\right)^{2}\right\rangle \simeq m .
$$

Taking into account the definition of an average number of hops per exciton,

$$
m=\langle i\rangle=\sum_{i=1}^{n}\left[i P_{i}(t)\right] / \sum_{i=0}^{n} P_{i}(t),
$$

and substituting Eqs. (6)-(8) into Eq. (5), one obtains 


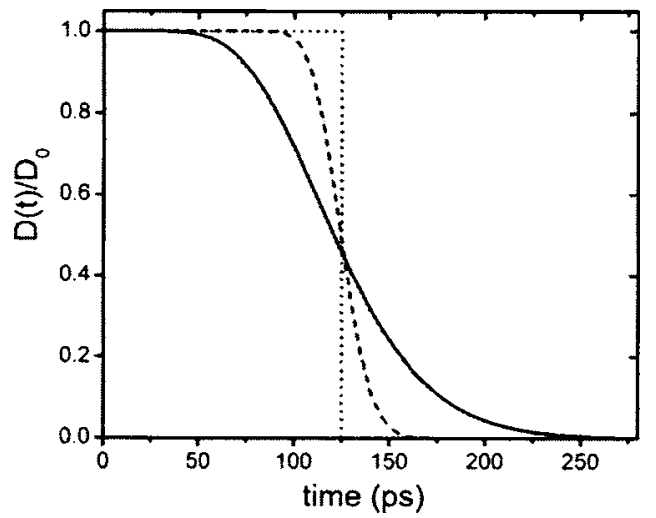

FIG. 3. Time-dependent diffusion coefficient for excitons as calculated using Eq. (6) for $n=10$ (solid line), $n=100$ (dashed line), and $n=\infty$ (dotted line).

$$
\begin{aligned}
D(t) & =\frac{\lambda^{2}}{2} \frac{d}{d t}\left[\sum_{i}^{n}\left\{i P_{i}(t)\right\} / \sum_{i}^{n} P_{i}(t)\right] \\
& =\frac{\lambda^{2}}{2}\left[\left\{\sum_{i=0}^{n-1} \frac{i-\gamma t}{(i-1) !} \gamma^{i} t^{t-1} e^{-\gamma t}\right\}+\frac{n}{(n-1) !} \gamma^{n} t^{n-1} e^{-\gamma t}\right] .
\end{aligned}
$$

According to Eq. (9) the characteristic time scale $t_{l o c}$ for exciton localization depends on both the number of energy levels, $n$, per lattice cell and an exciton hopping rate $\gamma$. The time-dependent diffusion coefficient calculated using Eq. (9) for different values of $n$ and a constant $t_{l o c}=n / \gamma$, suggesting that $\gamma=n / t_{l o c}$, is shown in Fig. 3. Calculations show that $D(t)$ changes from its maximum value to zero steeper if $n$ increases. Such a dependence of $D(t)$ upon $n$ is due to the fact that the exciton distribution becomes narrower with increasing $n$. The half-width of the exciton distribution $\Delta n_{1 / 2}$ in the space of the energy level numbers derived from Eq. (4) using Stirling's formula is $\Delta n_{1 / 2} \simeq 2 \sqrt{\gamma t \ln 2}$. In the space of exciton energies the distribution half-width is

$$
\delta E_{1 / 2} \simeq \Delta n_{1 / 2} \Delta E / n=2 \Delta E \sqrt{\frac{t \ln 2}{t_{l o c} n}},
$$

indicating a narrowing with increasing $n$.

At the interface with an inorganic oxide the excitons almost immediately dissociate by injecting electrons into the oxide. Indeed, it has been reported that the electron transfer reaction occurs in less than $100 \mathrm{fs} .{ }^{12}$ The value of $w_{j, i}$ can then be derived taking into account all possible exciton trajectories to the interface (one example of such trajectory is shown in Fig. 2 with solid arrows):

$$
\begin{aligned}
w_{j, i}= & 1-\theta(i-j)(1 / 2)^{j}-\theta(i-2-j) j(1 / 2)^{j+2}-\theta(i-4-j) \\
& \times j\left(\sum_{k=2}^{J+1} k\right)(1 / 2)^{j+4}-\theta(i-6-j) j\left(\sum_{k=3}^{J+2} \sum_{k_{1}=2}^{k} k_{1}\right)(1 / 2)^{j+6} \\
& -\theta(i-8-j) j\left(\sum_{k=4}^{J+3} \sum_{k_{1}=3}^{k} \sum_{k_{2}=2}^{k_{1}} k_{2}\right)(1 / 2)^{j+8}
\end{aligned}
$$

$$
-\cdots-j \theta(i-2 n-j)\left(\sum_{k=n+1}^{j+n} \cdots \sum_{k_{m-1}=3}^{k_{m-2}} \sum_{k_{m}=2}^{k_{m-1}} k_{2}\right)(1 / 2)^{i} \text {, }
$$

where $n=$ integer $[(i-j) / 2], \theta(l)=1$ if $l \geqslant 0$ and $\theta(l)=0$ otherwise, and the factor $(1 / 2)$ indicates an equal probability for an exciton to hop forward and backward.

The analysis given for the one-dimensional system can be applied to two- and three-dimensional systems $(\operatorname{Dim}=2,3)$ by taking into account that transport in higher dimensions consists of independent transport in two or three orthogonal directions. To use the previous results the hopping probability $\gamma$ and the total number of exciton energy levels, $n$, per lattice site in Eqs. (4) and (9) should be replaced with $\gamma /$ Dim and an effective number $n_{k}$ of maximum hops in one independent direction, respectively. The factor of $1 / 2$ and number of hops $i$ in Eq. (11) should be replaced with 1/(2 Dim) and an effective number of hops $i_{k}$ in the direction of interface. In a more general case of rough interfaces properly modified for higher-dimensional systems Eq. (1) with appropriate boundary conditions could be solved numerically using ordinary finite difference methods on a square (Dim $=2)$ or cubic $(\operatorname{Dim}=3)$ lattices.

\section{MODEL VERIFICATION}

The dependence of exciton diffusion coefficient upon the excitation energy given by Eq. (9) is verified by calculating the spectrum of internal quantum efficiency for an excitonic solar cell based on a $\mathrm{MEH}-\mathrm{PPV} / \mathrm{TiO}_{2}$ composite. The first step would be to determine exciton transport parameters by fitting time- dependent photoluminescence (PL) intensity $I_{\mathrm{PL}}$ of excitons in MEH-PPV.

\section{A. Experimental setup for studies of exciton PL in MEH-PPV and APCE of the composite solar cells}

Time-resolved photoluminescence measurements were performed in the vacuum at residual pressures lower than 0.1 mbar to avoid photo-oxidation. No sample degradation was observed during repeated measurements. The time evolution of the luminescence was measured with a Hamamatsu streak camera using the second harmonic of a Ti:sapphire laser at $3.1 \mathrm{eV}$ with pulse width of $100 \mathrm{fs}$ for excitation at a pulse repetition rate of $82 \mathrm{MHz}$. The devices tested here have a 50-100-nm-thick MEH-PPV layer spin coated from chlorobenzene solution onto a $2-\mu \mathrm{m}$-thick sintered nanocrystalline $\mathrm{TiO}_{2}$ film deposited on an ITO/glass electrode. A 50 -nm-thick Au contact was evaporated onto the polymer film to complete the device. ${ }^{13}$

An incident photon-to-conducting-electron conversion efficiency (IPCE, external quantum efficiency of the devices) was measured in vacuum (at $1 \mathrm{~Pa}$ pressure) under illumination with a halogen lamp using the Keithley 2400 Digital Source Meter equipped with a monochromator. During each measurement an Ocean Optics USB2000 spectrometer calibrated with a standard tungsten halogen light source was simultaneously engaged to measure light illumination inten- 


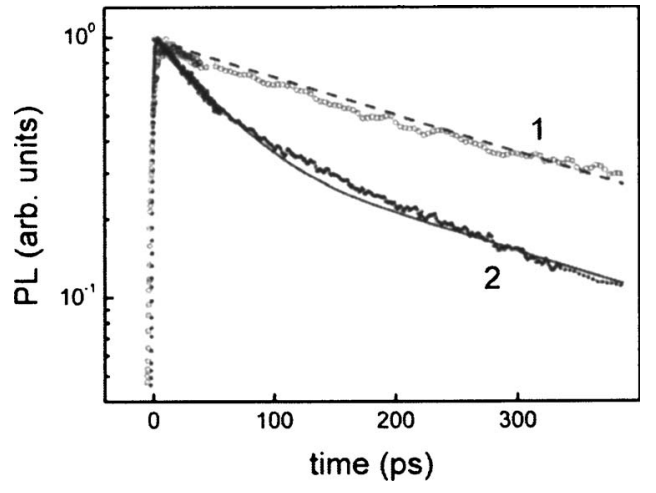

FIG. 4. PL intensity as a function of time in logarithmic scale. The symbols are experimental data for MEH-PPV film deposited on quartz (1) and $\mathrm{TiO}_{2}$ (2) substrates, respectively. The dashed curve corresponds to monoexponential decay enabling determination of exciton lifetime $\tau$. The solid curve is calculated using Eq. (9) for $n=10$ and $\gamma, 8 \times 10^{10} \mathrm{~s}^{-1}$.

sity using a partial mirror. The temperature of the sample was controlled by a thermocouple to confirm that any heating effect imposed by light absorption was negligible. The APCE was calculated taking into account light reflection at each interface and light absorption in each internal layer of the photovoltaic device, all measured with the Perkin-Elmer UV/ Vis absorption spectrometer.

\section{B. Comparison of theoretical and experimental results for PL and $\mathrm{APCE}$}

The $I_{\mathrm{PL}}(t)$ function, as shown in Fig. 4, decreases monoexponentially in the case of MEH-PPV deposited on a quartz substrate due to natural decay of excitons with the characteristic time constant $\tau \simeq 300$ ps. In contrast, the temporal behavior of $I_{\mathrm{PL}}(t)$ in the case of $\mathrm{TiO}_{2}$ substrate is highly nonmonoexponential; an initial fast decrease gradually slows down and approaches that observed in the case of quartz substrate. Such a time evolution of the PL intensity is due to exciton quenching at the interfaces with the $\mathrm{TiO}_{2}$ substrate. Indeed, the PL intensity is proportional to the overall number of excitons, and for the homogeneous initial distribution of photogenerated excitons it is given by

$$
I_{\mathrm{PL}}(t) \propto \sum_{j, i} w_{j, i} P_{i}(t) .
$$

Estimating the average hopping distance for excitons $\lambda$, $1 \mathrm{~nm}$, and the effective number of exciton energy levels, $n$ $=\Delta E / \varepsilon \simeq 10\left(\varepsilon\right.$ is the Stokes shift $\left.{ }^{14}\right)$, we determined the value of $\gamma \simeq 8 \times 10^{10} \mathrm{~s}^{-1}$, by fitting the experimental curves with the model ones. It turns out that the model is not very sensitive to the value of $n$ itself, but to the ratio $\gamma / n$, which represents the rate of exciton relaxation through the energy scale.

The APCE spectrum for a solar device with a given polymer layer thickness $d=m \lambda$ and illumination photon energy $h \nu$ is

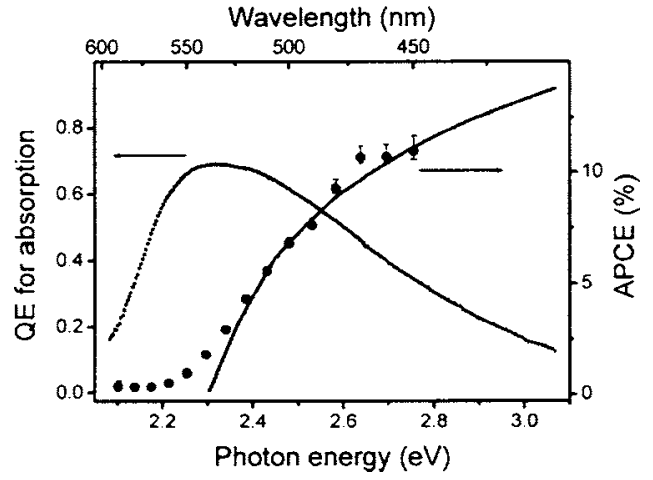

FIG. 5. APCE as a function of incident photon energy obtained experimentally (solid circles) and theoretically (solid line). The theoretical curve is divided by the factor 3.95 to account for possible decrease in APCE due to transport of charge carriers. The quantum efficiency (QE) of light absorption, a fraction of photons absorbed within 50-nm-thick MEH-PPV with respect to the incident photons onto a device is also plotted which shows the photoharvesting ability of the device.

$$
\operatorname{APCE}(h \nu) \sim \int_{0}^{\infty}\left\{e^{-1 / \tau}-\frac{1}{m} \sum_{j, i} w_{j, i} P_{i}(t)\right\} d t / \int_{0}^{\infty} e^{-1 / \tau} d t .
$$

As can be seen from the expression in brackets, the higher the PL intensity for the excitons [given by Eq. (12)], the lower will be the APCE, because fewer excitons inject their electrons into the inorganic oxide. An experimental APCE spectrum was determined using measured photocurrent and photoabsorption quantum efficiency evaluated by light transmission measurements. The obtained APCE spectrum is shown in Fig. 5. The experimental curve is scaled by a factor of 3.95 , the procedure actually taking into account the influence of charge transport losses on the device efficiency. The shape of the simulated curve fits well to the experimental data except for the energy domain around the light absorption edge at $2.4 \mathrm{eV}$, at which our assumption of a boxcar shape of the exciton DOS would be a too rough approximation. The observed increase in the APCE with excitation light energy indicates that an increased number of excitons reach organic-inorganic interfaces contributing to the charge separation in the solar cell. This suggests that excitons excited on higher-energy levels travel longer, as they have a higher effective number of exciton energy levels, $n$, to pass before reaching localization level. Using the definition of $D(t)$ given by Eq. (9) one can characterize exciton transport by the diffusion length $L$ defined as an average distance the exciton travels over its lifetime $L=\left(2 \int_{0}^{\tau} D(t) d t\right)^{1 / 2}$, which in the case of MEH-PPV gives the value $\sim 20 \mathrm{~nm}$.

\section{CONCLUSIONS}

We have developed a simple and powerful model to describe exciton transport in disordered systems, such as conjugated polymers. The model takes into account resonance energy transfer and inhomogeneous broadening of exciton energy levels due to structural disorder. In the cases when the 
system geometry is simple enough-i.e., homogeneous disordered systems or composite systems with flat interfacesthe model allows an analytical solution. In the cases of more complicated geometry the model allows efficient numerical simulations of exciton transport. Parametrization of the model performed using time-resolved PL data allowed predicting the internal quantum efficiency of real excitonic solar device based on $\mathrm{MEH}-\mathrm{PPV} / \mathrm{TiO}_{2}$ nanostructure. The predicted APCE value is based solely on exciton transport prop- erties and can be used to identify the contribution to the internal quantum efficiency arising from the charge transport losses in the device.

\section{ACKNOWLEDGMENT}

This work is funded by the Toppan Printing Co. Ltd. (Tokyo, Japan).
${ }^{1}$ I. D. W. Samuel, G. Rumbles, C. J. Collison, R. H. Friend, S. C. Moratti, and A. B. Holms, Synth. Met. 84, 497 (1997).

${ }^{2}$ M. Granstrom, K. Petritsch, A. C. Arias, A. Lux, M. R. Andersson, and R. H. Friend, Nature (London) 395, 257 (1998).

${ }^{3}$ B. Mollay, U. Lemmer, R. Kersting, H. Kurz, E. O. Göbel, H. Bässler, and H. F. Kauffmann, Phys. Rev. B 50, 10769 (1994).

${ }^{4}$ O. Mirzov, F. Cichos, C. von Borczyskowski, and I. G. Scheblykin, Chem. Phys. Lett. 386, 286 (2004).

${ }^{5}$ A. Ruseckas, M. Theander, L. Valkunas, M. R. Andersson, O. Inganäs, and V. Sundström, J. Lumin. 76\&77, 474 (1998).

${ }^{6}$ M. Scheidler, U. Lemmer, R. Kersting, S. Karg, W. Riess, B. Cleve, R. F. Mahrt, H. Kurz, H. Bässler, E. O. Göbel, and P. Thomas, Phys. Rev. B 54, 5536 (1996).

${ }^{7}$ W. F. Pasweer, P. A. Bobbert, and M. A. J. Michels, Prog. Solid State Chem. 1, 164 (2003).

${ }^{8}$ B. Movaghar, M. Grünewald, B. Ries, H. Bässler, and D. Würtz, Phys. Rev. B 33, 5545 (1986).
${ }^{9}$ H. Cordes, S. D. Baranovskii, K. Kohary, P. Thomas, S. Yamasaki, F. Hensel, and J.-H. Wendorff, Phys. Rev. B 63, 094201 (2001).

${ }^{10}$ D. Beljonne, G. Pourtois, C. Silva, E. Hennebicq, L. M. Herz, R. H. Friend, G. D. Scholes, S. Setayesh, K. Müllen, and J. L. Brédas, Proc. Natl. Acad. Sci. U.S.A. 99, 10982 (2002).

${ }^{11}$ B. J. Schwartz, T. Q. Nguyen, J. J. Wu, and S. H. Tolbert, Synth. Met. 116, 35 (2001).

${ }^{12}$ N. A. Anderson, E. C. Hao, X. Ai, G. Hastings, and T. Q. Lian, Chem. Phys. Lett. 347, 304 (2001).

${ }^{13}$ M. J. Carey, V. M. Burlakov, B. M. Henry, K. R. Kirov, G. R. Webster, H. E. Assender, G. A. D. Briggs, P. L. Burn, and C. R. M. Grovenor, Proc. SPIE 5215, 32 (2004).

${ }^{14}$ N. T. Harrison, D. R. Baigent, I. D. W. Samuel, R. H. Friend, A. C. Grimsdale, S. C. Moratti, and A. B. Holmes, Phys. Rev. B 53, 15815 (1996). 